\title{
Calcific stenosis of a mitral valve xenograft in a patient in chronic renal failure
}

\author{
MARTIN J RAFTERY, GEOFF KOFFMAN, J STEWART CAMERON \\ From the Renal Unit, Guy's Hospital, London
}

SUMMARY A 61 year old man presented with mitral regurgitation and glomerulonephritis caused by suspected infective endocarditis. His glomerulonephritis remitted on immunosuppression but mitral valve replacement with a Carpentier-Edwards porcine xenograft later proved necessary. The patient became dialysis dependent and cadaveric renal allografting was complicated by delayed graft function and refractory pulmonary oedema. Cardiac catheterisation showed severe calcification and stenosis of the xenograft with a transvalvar gradient of $23 \mathrm{~mm} \mathbf{~ H g}$ and despite emergency valve replacement the patient died. The valve had been in place less than five years. The rapid calcification and stenosis of a bioprosthetic heart valve calls into question the use of such prostheses in patients with chronic renal failure.

Valvar heart disease is a common complication of end stage renal failure and the lesions most commonly encountered are mitral annular calcification and aortic valve calcification. ${ }^{12}$ The incidence of these lesions increases with length of time on dialysis and when there is poor control of plasma concentrations of phosphate and calcium. ${ }^{23}$ They are rarely associated with haemodynamically important stenosis and they may cause tachycardia and bradycardia. We have recently encountered massive calcific excrescences on the supporting structure of a mitral valve xenograft that had been in place for less than five years in a patient with chronic renal failure. The calcification caused tight mitral stenosis but no regurgitation and resulted in a complicated clinical course after renal transplantation. Finally it caused the patient's death.

\section{Case report}

A 61 year old man was referred with oedema, episodes of severe dyspnoea, and palpitation. He had normochromic, normocytic anaemia; a pansystolic murmur compatible with mitral regurgitation; haematuria; proteinuria; and a plasma creatinine of $557 \mu \mathrm{mol} / 1$ (6.9 mg/dl). Echocardiography showed left atrial enlargement and thickened mitral cusps without visible vegetations. Despite numerous negative blood cultures a diagnosis of infective endocarditis and immune-complex mediated

Requests for reprints to Dr Martin J Raftery, Renal Unit, Guy's Hospital, London SE1 9RT. glomerulonephritis was made. A renal biopsy specimen showed a focal proliferative glomerulonephritis with crescent formation in $80 \%$ of glomeruli. Serum concentrations of complement were normal and the patient was treated with a combination of diuretics, antibiotics, corticosteroids, and azathioprine. His oedema subsided and his renal function improved such that his plasma creatinine fell to $250 \mu \mathrm{mol} / 1(2.8 \mathrm{mg} / \mathrm{dl})$.

Dyspnoea worsened one year later and cardiac catheterisation showed severe mitral regurgitation, trivial aortic regurgitation, and normal coronary arteries. The mitral valve was replaced with a $31 \mathrm{~mm}$ Carpentier-Edwards porcine xenograft. The resected mitral valve had a ruptured posterior cusp and there was histological evidence of endocarditis. Although no organisms were grown from the resected material, a six week course of gentamicin and erythromycin was prescribed. The patient made an uneventful recovery and anticoagulants were stopped after three months. Renal function gradually declined, however, and the patient became dialysis dependent two years after initial presentation. Persistent hypercalcaemia associated with a high serum concentration of parathormone was noted and tertiary hyperparathyroidism was diagnosed. This resolved after total parathyroidectomy in January 1986.

In April 1986 cadaveric renal transplantation was performed and a mid-distolic murmur was heard before operation but there was no associated pansystolic murmur. After operation the renal allograft did not function immediately and the patient experien- 
ced repeated episodes of pulmonary oedema. Despite vigorous dialysis and ultrafiltration, pulmonary oedema persisted and echocardiography showed that the mitral valve prosthesis was echogenic, heavily calcific, and poorly mobile. Cardiac catheterisation confirmed severe mitral stenosis with a transvalvar gradient of $23 \mathrm{~mm} \mathrm{Hg}$ but there was no evidence of regurgitation. An emergency mitral valve replacement was performed and at operation the cusps of the prosthesis were intact and there was no evidence of endocarditis. There were exuberant calcific outgrowths from the points of cusp attachment to the supporting structures, such that valve opening was severely impaired. The bioprosthesis was removed and replaced by a $29 \mathrm{~mm}$ Björk-Shiley tilting disc prosthesis. The postoperative course was complicated by hypotension, inotrope dependence, and an inability to wean the patient from assisted ventilation. The patient died 16 days postoperatively.

\section{Discussion}

Calcification of the mitral annulus is a common finding in the elderly at necropsy but may be present at a much earlier age in dialysis dependent patients. ${ }^{24}$ It is rarely of haemodynamic importance, but mitral stenosis secondary to massive annular calcific deposits associated with a small hypertrophied left ventricle has been described. ${ }^{5}$ The premature annular calcification in dialysis dependent patients has been associated with raised plasma concentrations of phosphate, the consequent raised calciumphosphate product, and the length of time on dialysis. ${ }^{23}$ An increased incidence of previous parathyroidectomy has also been noted.

We describe a dialysis dependent patient who was given a mitral valve xenograft because of mitral regurgitation thought to be caused by culture negative endocarditis. Prosthetic valve function seemed to be normal for over four years but a middiastolic murmur was heard before renal transplantation. The patient was at increased risk of dystrophic calcification because he had hyperphosphataemia caused by renal failure and hypercalcaemia caused by tertiary hyperparathyroidism while on dialysis. The diagnosis of prosthetic valve dysfunction was delayed because there was no regurgitant murmur and because it was assumed that pure stenosis was unlikely. The pattern of calcification found at operation explained the findings at auscultation in that the cusps were spared but exuberant calcification of the supporting structures prevented cusp excursion and interfered with valve opening. The consequence was a three week period of recurrent pulmonary oedema and delayed graft function caused at least in part by the low cardiac output produced by the tight stenosis.
The cause of the severe calcification of the bioprosthesis is not established but we presume that the intermittent hyperphosphataemia and the period of sustained hypercalcaemia in the year before transplantation were at least partly responsible. Early degeneration of a mitral valve bioprosthesis has previously been described in a patient taking self prescribed calcium supplementation but without documented hypercalcaemia. ${ }^{6}$ There have also been reports of early calcification of bioprosthetic valves and early failure in children and adolescents that was ascribed to increased calcium metabolism associated with growth; and one dialysis dependent patient has been described in whom there was early failure of both mitral and aortic bioprostheses. ${ }^{7}$

It seems likely that the risk factors for mitral annular calcification and aortic valve calcification in dialysis dependent patients are the same for bioprosthetic xenografts in the same population. ${ }^{2}$ Soft tissue calcification, particularly of blood vessels, is extremely common in dialysis dependent patients and because many of these patients are living longer, many are now being referred for coronary artery bypass grafts and valve replacements. The placing of bioprosthetic material in the circulation of such patients is likely to provoke dystrophic calcification and we believe that the case we describe should lead to the reconsideration of the use of bioprosthetic heart valves in dialysis dependent patients.

\section{References}

1 Forman MB, Virmani R, Robertson RM, Stone WJ. Mitral annular calcification in chronic renal failure. Chest 1984;85:367-71.

2 Maher ER, Young G, Smythe-Walshe B, Pugh S, Curtis JR. Aortic and mitral valve calcification in patients with end-stage renal failure. Lancet 1987;ii:875-7.

3 Nestico PF, Depace NL, Kotler MN, et al. Calcium phospherous metabolism in patients with and without atrial annular calcium. Am J Cardiol 1983;51: 497-500.

4 Fulkerson PK, Beaver BM, Anseon JC, Graber HL. Calcification of the mitral annulus: etiology, clinical ov associations, complications and therapy. Am J Med 1979;66:967-77.

5 Hammer WJ, Roberts WC, de Leon AC. Mitral stenosis secondary to combined massive mitral annular calcific $\sigma$ deposits and small hypertrophied left ventricles. $A m J$ Med 1978;64:371-6.

6 Moront MG, Katz NM. Early degeneration of a porcine aortic valve prosthesis in the mitral valve position in an elderly woman and its association with long term calcium carbonate therapy. Am J Cardiol 1987; 59:1006-7.

7 Schoen SJ, Collins JJ, Cohn LH. Long term failure rate $\mathbb{Q}$ and morphologic correlations in porcine bioprosthetic heart valves. Am J Cardiol 1983;51:957-64. 